\title{
Digital Rurbanization: decolonizing Fab Labs. The case study of Fab Lab Austral in Puerto Williams, Chile
}

\author{
Tomás Vivanco Larrain \\ Pontificia Universidad Católica de Chile | Chile | tvivancol@uc.cl \\ Tongji University | China | \\ Philip Yuan \\ Tongji University | China | philipyuan007@tongji.edu.cn
}

\begin{abstract}
Global digital infrastructures enable rural settlements to link their communities, local and natural resources with digital production capacities, generating digital urbanization processes. This article develops the concept of Digital Rurbanization presenting the coupling process of Fab Lab Austral, the southernmost Fab Lab in the world, with its community, ecosystem and global infrastructures. Producing a decolonized process of distributed design and making of meaningful prototypes with digital fabrication tools and methods, contributing to its sustainable development and autonomy based on technological education for the production of local solutions and social capital.
\end{abstract}

Keywords: Fab Labs; Digital Urbanization; Fab Communities; Digital fabrication; Decolonizing Design

\section{INTRODUCCIÓN}

Las capitales de los países latinoamericanos han experimentado importantes transformaciones urbanas, buscando integrarse a circuitos de ciudades globales (Sassien, 2010), generando importantes contrastes entre las capitales o grandes ciudades con ciudades o poblados rurales y aislados. Gracias a sus particulares contextos, algunos de estos asentamientos tienen la potencia de convertirse en modelos globales de desarrollo sustentable, indicando un eventual camino a seguir para el resto de las ciudades rurales.

Las tecnologías digitales de comunicación y fabricación juegan un rol clave para iniciar nuevos procesos de urbanización, basados en la transferencia e implementación de soluciones tecnológicas. Cambiando el modelo de "colonización industrial convencional", por uno sustentable, colaborativo con tecnologías de fabricación flexibles y locales (Diez, et. al, 2018).

Este artículo presenta el proceso de transición tecnológica de la comunidad de Puerto Williams Chile, por medio de procesos de urbanización digita. Describiendo las primera dos etapas de implementación del Fab Lab Austral, el laboratorio de fabricación digital mas austral del mundo, que opera como un centro de innovación comunitario abierto, contribuyendo con el desarrollo sustentable, articulación social y de transformación educacional. Enfocado en procesos digitales por sobre los productos tecnológicos.

\section{CONTEXTO}

Puerto Williams, se ubica en la Isla de Navarino, en la comuna de Cabo de Hornos, Chile, siendo la ciudad mas austral del mundo. Fue fundado en 1953 como una base de la Armada Chilena, hoy tiene una población cercana a los 2.200 habitantes, de la cual aproximadamente 550 son niños. Su estructura social esta compuesta por miembros de la Armada, antiguos habitantes, nuevos habitantes y la comunidad del pueblo originario Yagán.

La industria pesquera de cultivo y pesca de Centollas es la actividad económica principal, seguida por los servicios públicos. Dado su importante rol geopolítico, su potencial turístico y ecosistema natural, durante los últimos años el gobierno de Chile ha impulsado una serie de inversiones y planes para consolidar a Puerto Williams como ciudad estratégica, junto con impulsarla como un polo de turismo mundial.

Gracias a sus corrientes, ecosistema, su condición insular $y$ al hecho de que se encuentra al fin de la cadena de suministro mundial, Cabo de Hornos es un laboratorio natural que se ha logrado mantener aislado de la era industrial (Verbeck, 2016), desafectándola de las consecuencias de la globalización. Por ello, en el año 2005, Cabo de Hornos recibió el reconocimiento como Reserva Mundial de la Biósfera por la UNESCO, y su agua es catalogada como una de las mas puras del mundo (University of North Texas, 2016). 
Sin embargo, esta condición de aislación ha expuesto a Puerto Williams a amenazas ambientales y productivas, ya que todas las necesidades básicas como materias primas, alimentos, e incluso la energía, son importadas por barco. A la vez, la inexistencia de infraestructuras, modelos o planes de reciclaje o reutilización de materias, generando una acumulación continua de residuos.

Por otra parte, Puerto Williams es una de las pocas ciudades de Chile que no cuenta con conexión a internet de alta velocidad, para su acceso, se deben dirigir señales desde Argentina a muy baja potencia. Se espera que el proyecto Fibra Óptica Austral, encuentra canalizado y conectado con el continente, esté operativo a finales del año 2020. Paralelamente en enero del año 2019 se inauguró el Fab Lab austral perteneciente a la red mundial de Fab Labs impulsada por le MIT que cuenta con mas de 1.900 laboratorios extendidos en todo el mundo, que cuenta Como complemento a este equipamiento digital global, el proyecto Fibra Óptica Austral se encuentra canalizado y conectado con el continente, el cual se espera que esté operativo a finales del año 2020.

\section{LA ERA URBANA: INDUSTRIALIZACIÓN DE LO RURAL}

Para que las ciudades puedan ser ciudades, manteniendo su fenómeno urbano en el tiempo, requieren de un soporte territorial y material que se extiende por sobre los límites urbanos, dotando a territorios - aparentemente apartados o rezagados de procesos de urbanización - de equipamiento físico y espacial como servicios para que estas operen. Este fenómeno definido como el crecimiento exponencial de las ciudades (Brenner, 2016) que describe el fenómeno global (Sassen, 2006) de las ciudades en donde prácticamente todo el territorio del planeta se pone a disposición del funcionamiento y crecimiento de las ciudades.

Esta perspectiva de comprensión de la era urbana, a partir de los procesos de industrialización, permite cuantificar el impacto ambiental de la globalización de las ciudades sobre la extensión del territorio, que han moldeado no solo la manera en como producimos los objetos y bienes, sino que también han gatillado en los grandes procesos migratorios del campo hacia las ciudades, transformando una economía basada en la agricultura por una basada en la industrialización, producción y consumo en masa (Jaune, 2006). En este proceso, constitutivo de las ciudades modernas, los individuos ya no son gobernados por la historia o por una determinada tradición, siendo atraídos por las oportunidades, productos y servicios que estas ofrecen. La hiperestimulación sensorial que la ciudad moderna ofrece desfragmenta el tiempo incitando al cambio constante y flujo de información como condiciones soportantes de la experiencia urbana.

La manufactura centralizada, con procesos tayloristas sumamente optimizados bajo el modelo de Ford es la respuesta directa a la necesidad del mercado de producir bienes de consumo a gran velocidad. Trayendo grandes contribuciones en los procesos ingenieriles, estandarizando componentes, procesos de manufactura y productos, teniéndose que diseñar nuevas máquinas y sistemas de calibración para que las líneas de producción pudieran ser continuas y perfectas. La velocidad y eficiencia de estas tareas requerían que los errores fuesen disminuidos al mínimo, forzando a los trabajadores a realizar solo una tarea de manera repetitiva optimizando su productividad y por consecuencia la producción.

Esto trae por consecuencia un desfase de velocidades entre la de la economía de las ciudades y la regeneración de los recursos naturales, generando un retraso en la relación acción/ reacción, en donde la complejidad socio territorial no es capaz de reaccionar a tiempo a las demandas de un mercado dinámico (Arboleda, 2015).

Los tiempos internos de un territorio soportado tanto en los recursos sociales como naturales no permiten la regeneración de estos, rompiendo las ecologías locales, obligando al mercado a buscar nuevos territorios (Urban Theory Lab, 2013) aumentando considerablemente la factura entrópica (Rifkin, 2009), rompiendo la estructura de soporte básico para el desarrollo de nuestra sociedad. La construcción de una sociedad mundo (Morin, 2010) nos ha llevado a la homogeneización de, entre otras cosas, la cultura, identidades, producción y economía. La economía capitalista, soportada en el modelo globalizado, ha sido desde su origen la economía de esta sociedad mundo, fracturando e imposibilitando el desarrollo de comunidades sustentables.

El territorio chileno, país con condiciones geográficas extremas y con una economía basada en la explotación y exportación de sus recursos naturales, junto con las industrias agroalimentaria y acuícola, puede ser comprendido como una gran infraestructura para las ciudades globales. Las mineras para la extracción de cobre en el Desierto de Atacama en el norte de Chile, los viñedos e industria agrícola en el centro y la industria acuícola en el sur, generan profundar alteraciones en el paisaje rural (Figura 1) racionalizándolo para optimizar la producción de recursos que serán consumidos en las ciudades.

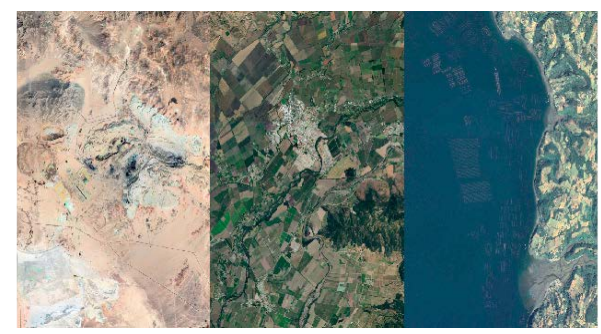

Figura 1: Racionalización productiva de territorio rural en Chile y el paisaje extractivo. Norte minería, centro vitivinícola, sur acuícola. (Google Earth, editada por el autor, 2020).

\section{LA ERA DIGITAL: DIGITALIZACIÓN DE LO RURAL}

Desde el primer cable submarino para el telégrafo atlántico en 1858, como de comunicación transoceánico entre Inglaterra y Estados Unidos, que redujo el tiempo de comunicación de 10 días a minutos, se ha sostenido un incremento en la construcción de infraestructuras de comunicación intercontinentales. Las que gracias a la implementación del proyecto Fibra Óptica Austral, se 
distribuye e ilumina con internet a todos los continentes (Figura 2), dando soporte a la conectividad digital global.

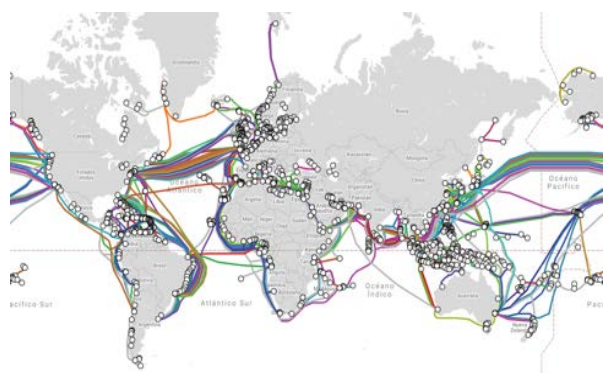

Figura 2: Mapa mundial del cableado submarino de Fibra Óptica (www.submarinecablemap.com, Equinix 2020).

La hiperconectividad y su impacto en nuestras vidas personales (Cheok, 2015), se han situado como medios de medios para la sociabilización y transferencia de información y comercialización, pero no se ha validado como una tecnología que permita resolver, por si sola, temáticas urbanas o sociales (Sassen, 2017). Es imposible para urbanistas y arquitectos resolver problemas muchos mas complejos que las mismas infraestructuras en si mismas son capaces de solucionar, de hecho, no hay necesidad de construir proyectos urbanos masivos para lograr impactar las ciudades, la idea de ciudades distribuidas (Vivanco 2017) está relacionada con la desmaterialización de las infraestructuras físicas para crear nuevos tipos de información y sistemas de comunicación (Castells, 1995), para centrarlas en las personas y en el valor colectivo.

A pesar de ello, la digitalización por medio de internet ha sido uno de los agentes claves para las reformas económicas a nivel global. China, por ejemplo, gracias al crecimiento exponencial del e-commerce con Taobao como plataforma de compra y venta, junto con Wechat y Alipay como plataformas de pago han dinamizado territorios que antes estaban invisibilidades por las lógicas urbanas convencionales. (Quartz, 2017). Las denominadas "Taobao Villages" se refiere a los pueblos rurales que tienen por lo menos 100 tiendas online o que el $10 \%$ de los propietarios de viviendas estén vinculados con el e-commerce, y donde se generan transacciones sobre el 1,5 millón de dólares utilizando Taobao como plataforma de transacción (Li, 2017).

Por otra parte, la Fab Lab Nertwork, red internacional de Laboratorios de Fabricación Digital, impulsada por el Center for Bits and Atoms del Massachussets Institute of Technology y la Fab Foundation, a logrado articular a mas de 1.900 laboratorios extendidos en todas las regiones del mundo. Poniendo a disposición de las ciudades y personas infraestructuras para la fabricación digital de "casi" cualquier cosa (Gershenfeld, 2019).

\section{PROTOTIPOS FÍSICOS EN LA ERA DIGITAL}

En este contexto digital, prototipar implica traspasar la barrera física de los objetos para generar conexiones simbólicas y significativas en ecosistemas locales. En este sentido, el diseño no orientado a objetos soporta la idea del diseño distribuido (Vivanco, 2017) que se relaciona con la desmaterialización de las infraestructuras y objetos físicos para crear nuevos tipos de relaciones basadas en los sistemas de comunicación e información (Castells, 1995), centrados en las personas y en su valor colectivo para la detección de oportunidades, diseño y producción de soluciones locales.

Diseñar en redes digitales implica tres condiciones relevantes que permiten crear una relación directa entre las personas, sus recursos disponibles, usuarios e infraestructuras digitales:

En primer lugar, las tecnologías digitales permiten la desintermediación, es decir, la supresión de intermediarios, generando oportunidades de fabricación bajo demanda y localmente, reduciendo considerablemente los costos económicos y energéticos añadidos a los objetos. Pudiendo conectar a creadores, usuarios y territorios de manera directa.

En segundo lugar, permite desagregar productos o procesos productivos, involucrando a nuevos actores y servicios. De este modo, soluciones complejas pueden ser resueltas de manera colaborativa y distribuida.

Por último, la digitalización fuerza a transformar productos físicos en digitales, desagrupando e individualizando la demanda, permitiendo a usuarios recibir productos en cualquier lugar y en cualquier momento.

La aplicación de lógicas digitales en territorios rurales o aislados permiten generar nuevas tipologías de transformaciones socio-territoriales en pos de la integración a redes globales de manera sustentable, conectadas con su entorno natural y complejidad social (Kotler, et. al,1984). Las nuevas tecnologías de la información nos permiten modificar el desfase temporal (Ibañez, Katsikis, 2014), reconectando demanda y oferta desde el territorio, reformulando el modelo capitalista convencional, reconectando a ciudadanos con productores y las capacidades productivas con las de regeneración ambiental (Ghosh, 2017) en las ciudades. Consolidándose un ecosistema centrado en las personas, soportado por un piso ecológico y social.

Los laboratorios, como centros abiertos y comunitarios para el diseño y producción de soluciones locales, por medio de proceso participativos (Hyysalo et al., 2014), permiten que personas sin requerir conocimiento previo puedan aprender y conectarse para explorar el desarrollo de potenciales necesidades locales y específicas.

\section{METODOLOGÍA}

El contexto aplicado en el caso de estudio plantea las siguientes preguntas de investigación y desarrollo ¿Cómo mejor la experiencia de habitar una ciudad extrema desde el desarrollo de prototipos en un laboratorio de fabricación digital? ¿puede una ciudad extrema y aislada del impacto productivo industrial, pero a la vez estar digitalmente conectada con el conocimiento y capacidades productivas globales.? ¿Cómo diseñar y preparar a una comunidad en la era pre-internet por medio de la digitalización como 
catalizador de la sustentabilidad por medio intervenciones de impacto?

La metodología de implementación del Fab Lab Austral se estructura en tres etapas de un año cada una (Figura 3), que comenzó el año 2019 junto con su inauguración con foco en el analfabetismo digital, la cual se expone en esta investigación, la segunda tiene foco en el desarrollo.

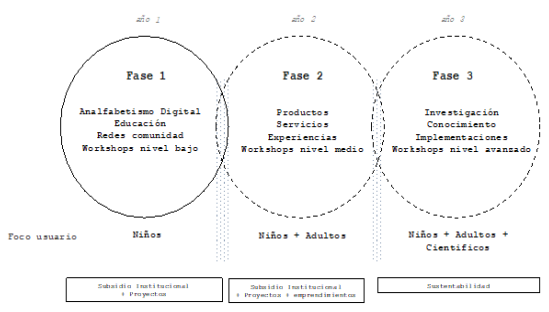

Figura 3: Metodología y etapas de implementación Fab Lab Austral (fuente propia, 2019).

\section{FAB LAB AUSTRAL}

Fab Lab as un Laboratorio de Fabricación Digital que gradualmente se ha convertido en un espacio de convergencia social para la comunidad local, la cual no cuenta con espacios comunitarios para el desarrollo de proyectos, producción, experimentación y educación. Operando como un centro de creatividady producción local y autónomo, al fin de la cadena de suministro, con foco en las comunidades, ecosistemas, ciencias, artes, y tecnologías digitales. Creando una cultura digital basada en la formación de personas, sustentabilidad, prototipado e investigación. Vinculando el quehacer y necesidades locales con infraestructuras y redes globales.

El desarrollo de actividades se enmarca en la pregunta ¿Qué pasaría si se reducen las importaciones de materias primas y productos que viajan desde el continente por barco, y se aumenta la transferencia digital de objetos distribuidos por la fibra óptica para ser fabricados con recursos locales?

Esta pregunta el desafío de catalizar procesos de urbanización digital por medio de la transferencia de conocimiento, aprendizaje local, investigación y prototipado, como motores y objetivos en si mismos.

\section{TRANSFERENCIA DE CONOCIMIENTO Y CONOCIMIENTO LOCAL}

Con el fin de acortar las brechas de analfabetismo digital, junto con generar conciencia sobre el valor de la fabricación digital de manera transversal en la comunidad de Puerto Williams, durante la primera fase de implementación el foco se centró en la transferencia de conocimiento a niños y docentes del Liceo Donald Mc Intyre Griffiths, único establecimiento educacional. De este modo se construye una masa crítica, generando un capital social que permita asentar las bases del laboratorio en la comunidad.
Para impartir los contenidos, se diseñó un programa de talleres organizados en una taxonomía de habilidades estructurados según objetivos de aprendizaje y masa crítica de interés, donde se impartieron 25 talleres donde participaron 280 asistentes. Cada tecnología fue enseñada por medio de un método de diseño comprensible sin que requiera de conocimientos previos en modelado computacional, junto con vincularla con algún dolor o necesidad de la isla Navarino. Por ejemplo, la impresión 3D fue enseñada por medio del escaneo tridimensional de objetos y su impacto fue relacionado con el reciclaje de plástico como potencial insumo.

Gradualmente, los distintos grupos sociales de la comunidad de Puerto Williams, articulados por el Liceo, fueron acercándose al laboratorio con el interés de desarrollar proyectos y prototipos para emprendimientos, en paralelo, representantes de organizaciones instituciones locales público y privadas organizaron sesiones se trabajo para el levantamiento de oportunidades y cocreación de soluciones (Figura, 4). Dando cuenta de la valoración del impacto transversal del laboratorio.

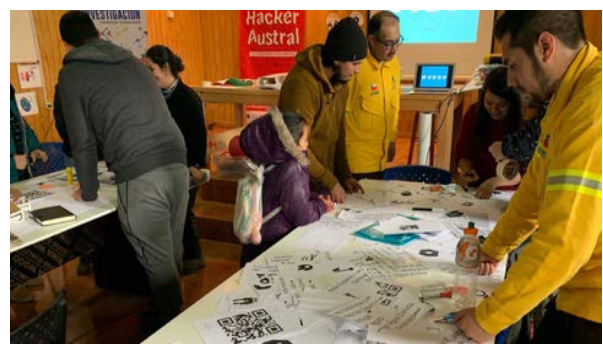

Figura 4: Taller de codiseño de la comunidad de Puerto Williams (fuente propia, 2019).

\section{CO-DISEÑO Y PROTOTIPOS DE SOLUCIONES LOCALES}

Mediante un proceso de codiseño entre los distintos actores de la comunidad, se identificaron recursos naturales como insumos para el desarrollo de soluciones tecnológicas en código abierto. Una de las principales oportunidades detectadas es la producción local de energía, y su transformación en sistemas de calefacción (Figura 5), junto con el desarrollo de bio materiales (Figura 6), benignos para el entorno.

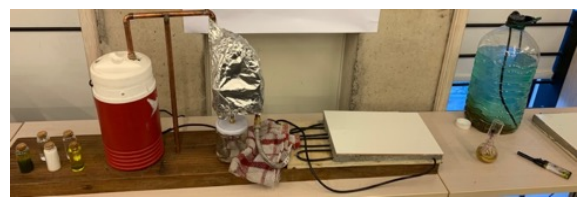

Figura 5: Sistema de calefacción casero en base a biodiesel a partir de algas locales. Prototipo desarrollado por estudiantes del Magíster en Diseño Avanzado de la Pontificia Universidad Católica de Chile (fuente propia, 2019). 


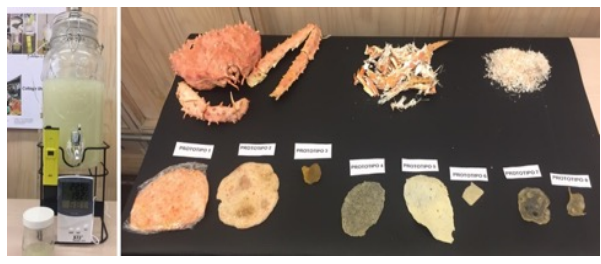

Figura 6: Sistema de extracción de quitina del caparazón de Centollas y prototipos de biocompuestos. Prototipos desarrollados por estudiantes del Magíster en Diseño Avanzado de la Pontificia Universidad Católica de Chile (fuente propia, 2019).

\section{RESULTADOS}

En esta primera fase, la brecha del analfabetismo digital fue acordada por medio de la transferencia de metodologías y conocimientos para convertir ideas en soluciones utilizando herramientas digitales. La idea de conectar Puerto Williams con el desarrollo tecnológico global generó un proceso de acoplamiento (Lee, Yoon, 2004) con la comunidad quienes produjeron una serie de tecnologías funcionales. Esta transferencia trajo como consecuencia el desarrollo de prototipos con base tecnológica se categoriza en dos ámbitos de intervención.

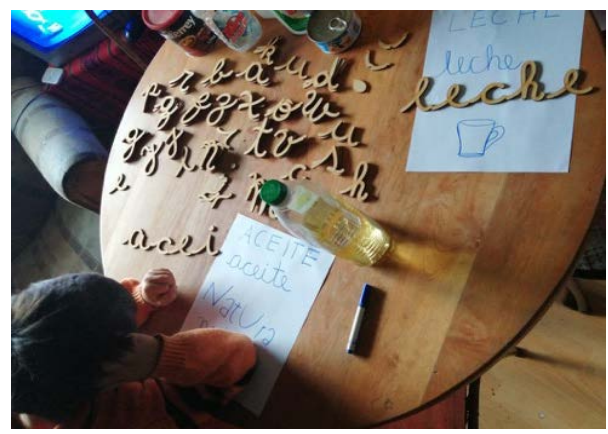

Figura 7: Escolar utilizando material didáctico en su hogar, producido por docente del Liceo Donald Mc Intyre (enviado por apoderado, 2020).

El primer ámbito se compuso de desarrollos para el apoyo a la educación de escolares y preescolares (Figura 7),

desarrollados en conjunto con profesores del Liceo quienes se iniciaban en la fabricación digital. En ellos, se puede apreciar un bajo desarrollo de técnicas o procesos digitales, mediante prototipos de baja resolución formal y tecnológica, sin procesos de postproducción (Figura, 8). Principalmente, los resultados se enfocaron en soluciones que permitieran resguardar el patrimonio cultural de la cultura Yagán, produciendo réplicas de canoas yaganes ensamblables, digitalizando objetos del muso Martín Gusinde, y material didáctico para complementar los procesos de educación en casa, situación obligatoria por la pandemia global.

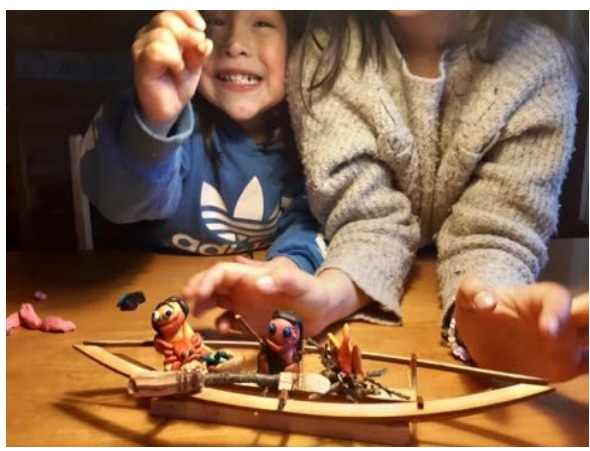

Figura 8: Canoa Yagán ensamblada por niños preescolares. Prototipo diseñado y fabricado por parvularias del Jardín Infantil Villa Ukika. (enviado por apoderado, 2020).

En este ámbito, los resultados técnicos formales quedan circunscritos en narrativas locales, las cuales dan cuenta de la estrecha relación entre los objetos y su ecosistema. La utilización de materiales disponibles localmente y la precisión de las formas expresan con lo mínimo el mensaje 0 relato que se quiere comunicar por medio del objeto como expresión cultural.

Por otra parte, el segundo ámbito de prototipos fue liderado por el equipo de diseñadores del Fab Lab Austral en conjunto con actores específicos de la comunidad, logrando resolver desafíos y necesidades que requerían de una mayor resolución técnica (Figura 9). Por ejemplo, durante la crisis sanitaria generada por el covid-19, se codiseñó, en conjunto con las enfermeras y médicos del hospital comunitario Cristina Calderón un escudo de protección facial para los trabajadores del hospital y una versión alternativa para los trabajadores de almacenes y tiendas expuestos a contacto directo con personas y clientes. También, en conjunto con bomberos, se adaptó y fabricó un filtro de aire, que se acopla a sus casos y mascarillas (Figura 10).

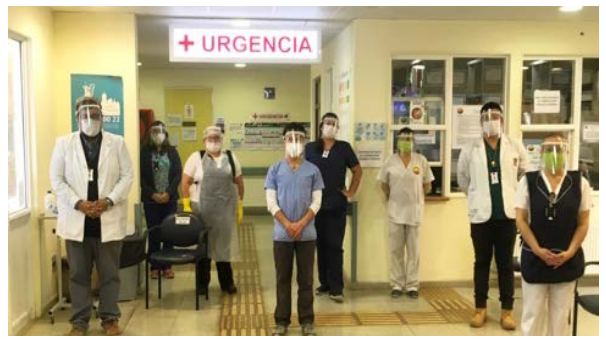

Figura 9: Trabajadoras del Hospital Comunitario Cristina Calderón con protectores faciales producido en Fab Lab Austral.

Ambos tipos de desarrollos dejan en evidencia el contraste entre el diseño conducido y direccionado por especialistas, versus el diseño emergente de la comunidad. Resaltando la idea de la estética como productor y regulador de experiencias (Tlostanova, 2017) y mediador del desarrollo cultural local por medio de objetos imperfectos para el experto. 


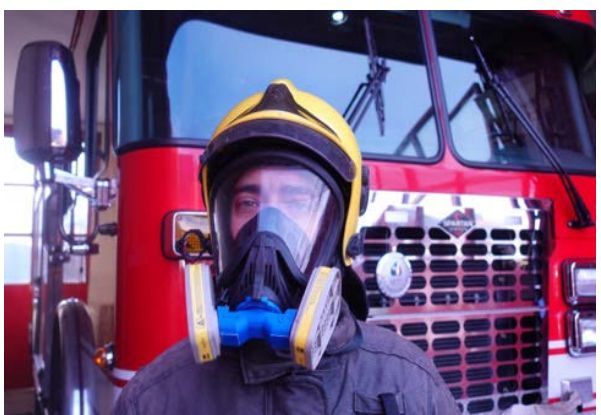

Figura 10: Bombero de Puerto Williams con filtro de aire adaptado.

\section{DISCUSIÓN}

Como primera fase de implementación, abordar el espacio de analfabetismo digital ha permitido dar acceso a la comunidad a equipamiento y conocimiento para producir ideas con tecnologías digitales. Esto a reducido la percepción de aislamiento del territorio, por cuanto la comunidad ha logrado producir sus propias soluciones de manera autónoma, generando un acoplamiento entre la comunidad (Lee, Yoon, 2004) y el Fab Lab Austral.

Transformar ideas en prototipos tangibles involucra la activación de procesos de diseño para productos artefactos y símbolos enmarcados en un contexto social, cultural, político y medioambiental. Esto genera un grado de significado en los individuos y las comunidades, transformado símbolos a productos, productos a servicios y servicios a sistemas (Buchanan, 2001), otorgando valor y capacidad de acción social.

El contraste estético- tecnológico de los prototipos realizado en el Laboratorio abren la reflexión sobre el impacto colonizador de los Fab Labs en territorios rurales, los cuales si se logran acoplar con su comunidad permiten el desarrollo de productos descolonizados y soberanos. Posibilitando del desarrollo creativo, tecnológico y dinámico de personas y comunidades (Tlostanova, 2017) no consideradas en los procesos de urbanización convencionales.

La conexión del Fab Lab Austral con sus recursos locales y comunidad ofrece una posibilidad única para que en el futuro cercano Puerto Williams pueda disminuir la cantidad de productos transportados físicamente por barco, aumentando los productos transferidos digitalmente por fibra óptica, para ser fabricados localmente. Estableciendo un modelo nuevo y replicable para la urbanización digital de asentamientos rurales remotos en Latinoamérica.

\section{REFERENCIAS}

Anthony H. F. Li, " E-commerce and Taobao Villages », China Perspectives [Online], 2017/3 | 2017, Online since 01 September 2017, connection on 28 October 2019. URL: http:// journals.openedition.org/chinaperspectives/7423

Arboleda, Martín. Financialization, Totality and Planetary Urbanization in the Chilean Andes. Geoforum 67, 2015.
Castells, Manuel. La ciudad informacional, Tecnologías de la información, reestructuración económica y el proceso urbanoregional. Alianza Editorial, 1995

Cheok, Adrian. Hyperconnectivity and the Future of Internet Communication. [online] http://www.city.ac.uk/news/2015/may/hyperconnectivity-andthe-future-of-internet-communication

Chilean National Census [online] https://www.censo2017.cl/

Diez, Tomás. Ed. Fab City: The Mass Distribution of (almost) anything. Institute for Advanced Architecture of Catalonia. 2018.

Dong-Seok Lee, Wan Chul Yoon, Coupling structural and functional models for interaction design, Interacting with Computers, Volume 16, Issue 1, February 2004, Pages 133161.

Ghosh, Swarnabh. Notes on Rurality or The Theoretical Usefulness of the Not-Urban. The Avery Review 27. 2017.

Ibañez, Daniel and Katsikis, Nikos. New Geographies 6: Grounding Metabolism. Harvard University Press, 2014.

Karana, E, Barati, B, Rognoli, V and Zeeuw van der Laan, A 2015, 'Material Driven Design (MDD): A Method to Design for Material Experiences', International Jour- nal of Design, 9(2), pp. 35-54.

Madina Tlostanova(2017) On decolonizing design, Design Philosophy

61, DOI: 10.1080/14487136.2017.1301017. Papers, 15:1, 51-

Morin, Edgar. ¿Hacia el abismo? Globalización en el siglo XXI. Ediciones Paidós, 2010.

Phillip M. Mach, Jaime L. Winfield, Roberto A. Aguilar, Kenneth C Wright, Guido F. Verbeck. A portable mass spectrometer study targeting anthropogenic contaminants in Sub-Antarctic Puerto Williams, Chile. International Journal of Mass Spectrometry, Volume 422. 2017, Pages 148-153.

Quartz, 2017. [online]: https://qz.com/899922/once-povertystricken-chinas-taobao-villages-have-found-a-lifeline-makingtrinkets-for-the-internet/

R. Buchanan. "Design Research and the New Learning." Design Issues, Vol. XVII, No. 4. 2001.

Rifkin, Jeremy. La civilización empática: La carrera hacia una conciencia global en un mundo en crisis. Tarcher Perigee, 2009.

Sampsa Hyysalo, Cindy Kohtala, Pia Helminen, Samuli Mäkinen, Virve Miettinen \& Lotta Muurinen. Collaborative futuring with and by makers, CoDesign. 2014.

Saskia Sassen. Embedded borderings: making new geographies of centrality, Territory, Politics, Governance. 2017.

Saskia, Sassen. Cities in a world economy. Thousand Oaks, 2006.Jaune, Mark. Cities and Consumption. Toutledge, 2006.

Sassen, Saskia La Ciudad Global: Nueva York, Londres, Tokio. Universitaria de Buenos Aires, 1999.

Tironi, M., Hermansen, P. Prototyping Multispecies Environments: attentiveness andF iction as modes of knowing. Design Research Society Conference 2018. 2018.

Urban Theory Lab-GSD (Neil Brenner, Nikos Katsikis, Danika Cooper, Ghazal Jafari). Visualizing an urbanized planetmaterials. in Neil Brenner (ed.), Implosions/Explosions: Towards a Study of Planetary Urbanization. Berlin: Jovis, 2013, 460-475

Vivanco, T. Design and Fiction as Instruments for Social Development. Distributed Design Book. Ediciones UC. 2016.

Vivanco, Tomás. Cybernetics infrastructures of quantitative and qualitative data infrastructures for distributed cities. Responsive Cities Conference, Barcelona 2017. 